\title{
Dislocation-Containing Planar Faults in Metastably Retained Hexagonal Barium Titanate
}

\author{
Yu-Chuan $\mathrm{Wu}^{\dagger, \dagger}$ \\ Department of Materials and Mineral Resources Engineering, National Taipei University of Technology, Taipei 10608, \\ Taiwan \\ Hong-Yang Lu*
}

Centre for Nanoscience, Institute of Materials Science and Engineering, National Sun Yat-Sen University,

Kaohsiung 80424, Taiwan

\begin{abstract}
Planar faults, designated $F_{1}-F_{6}$ and $F_{a}$, with intermixed dislocations in metastably retained hexagonal- $\mathrm{BaTiO}_{3}$ ceramics, were found and analyzed by transmission electron microscopy. Only faults with one, two, and four extra $c$-layers parallel to the basal plane have been identified. Fault vectors $\mathbf{R}_{F_{1}}=\mathbf{R}_{F_{a}}=$ $1 / 6[0221], \quad \mathbf{R}_{F_{2}}=\mathbf{R}_{F_{3}}=1 / 3[0 \overline{111}], \quad \mathbf{R}_{F_{4}}=1 / 3[\mathbf{0 1 1 2}], \quad$ and $\mathbf{R}_{F^{\prime}{ }_{1}}=\mathbf{R}_{F_{5}}=\mathbf{R}_{F_{6}}=\mathbf{R}_{F^{\prime}{ }_{a}}=1 / 6[0 \mathbf{0 2 2 1}]$ were determined adopting the $2 \pi g \cdot R=0$ (or $2 n \pi$ ) criteria in combination with highresolution imaging. Further, the embedded dislocations were half-partials with Burgers vectors $b=1 / 3\langle 1010\rangle$ determined by the $\mathbf{g} \cdot \mathbf{b}=\mathbf{0}$ effective invisibility criteria in conjunction with the eligible fault vectors. A rotation by $60^{\circ}$ about the $c$-axis was found between fault segments with $R_{F_{1}}=1 / 6[02 \overline{2} 1]$ and $R_{F_{1}^{\prime}}=$ $1 / 6[20 \overline{2} 1]$ located on either side of a partial. Basal dislocations with $b_{B}=1 / 3\langle 1210\rangle$ have dissociated into two prismplane Shockley half-partials with $b_{\operatorname{Pr}}=1 / 3\langle 1 \overline{100}\rangle$ by glide in the fault plane (0002) according to $1 / 3\langle 1210\rangle \rightarrow$ $1 / 3\langle 1 \overline{100}\rangle+1 / 3\langle 0 \overline{1} 10\rangle$. The fault segment $F_{1}^{\prime}$ encompassed by two half-partials is an extrinsic complex stacking fault.
\end{abstract}

\section{Introduction}

$\mathrm{H}$ IGH-TEMPERATURE ${ }^{1}$ hexagonal $(h-) \mathrm{BaTiO}_{3}\left(P 6_{3} / m m c\right.$, No. 194) have been retained metastably at room temperature with acceptor doping, ${ }^{2,3}$ for example, $\mathrm{Mg}^{2+}$ or $\mathrm{Fe}^{3+}$, substituting for the $\mathrm{Ti}^{4+}$ site, or by hot pressing ${ }^{4,5}$ in low oxygen partial pressures. The $6 \mathrm{H}$-polytype (i.e., $h-\mathrm{BaTiO}_{3}$ ) is not ferroelectric; another hexagonal phase $\left(P 2_{1}\right.$ (No. 17)) only becomes ferroelectric at $\sim 77 \mathrm{~K}\left(-196^{\circ} \mathrm{C}\right) .^{6}$ Although doped with acceptoroxide $\mathrm{Ga}_{2} \mathrm{O}_{3}, h$ - $\mathrm{BaTiO}_{3}$ has been explored ${ }^{7}$ for microwave dielectrics; unlike the tetragonal phase, its practical applications are still limited. Fault vectors $\left(\mathbf{R}_{F}\right)$ in the $h$ - $\mathrm{BaTiO}_{3}$ ceramics prepared by hot pressing in a graphite die assembly were analyzed and how these faults were generated in low oxygen partial pressures has been proposed..$^{5}$ It was suggested that the stacking faults described as interleaved $c-\mathrm{BaTiO}_{3}$ lamellae embedded in $h$ - $\mathrm{BaTiO}_{3}$ matrix are growth faults due to local oxygen excess accommodated by extra corner-sharing $c$-layer(s). The faults consisted of extra $c$-layers relative to the $(c h c)_{1}(c h c)_{2}$ (or equivalently (CBC)(ABA)) stacking sequence of $6 \mathrm{H}$-polytype. ${ }^{3,5}$ They contained no dislocations. ${ }^{5}$ Here, we report on an analysis con-

T. Mitchell—contributing editor centrating on planar faults, intermixed with dislocations, which have been divided into several segments. With dislocations running across the faults, the fault fringe contrast was distinctive on either side of a dislocation. A fringe contrast of alternate visibleinvisible bands in strong-beam images had appeared among the fault segments.

Eibl et $a .^{8}$ have reported the presence of both perfect and partial dislocations in $h-\mathrm{BaTiO}_{3}$ ceramics sintered at $1440^{\circ} \mathrm{C}$ in air, followed by annealing at $1400^{\circ} \mathrm{C}$ in an $\mathrm{N}_{2}-\mathrm{H}_{2}$ atmosphere. Similar to hexagonal close-packed metals (A3 hcp), and D $0_{19}$ intermetallics, the unit lattice translation vectors in $h-\mathrm{BaTiO}_{3}$ are $1 / 3\langle 11 \overline{2} 0\rangle,\langle 10 \overline{1} 0\rangle$, and [0001]. Perfect dislocations with $\mathbf{b}=$ $1 / 3\langle 11 \overline{2} 0\rangle$ and a pair of Shockley partials with $\mathbf{b}^{\prime}$ and $\mathbf{b}^{\prime \prime}=1 / 3\langle\overline{1} 100\rangle$, all lying in the basal plane, were identified. Dissociation of the former into a pair of Shockley half-partials, often found in hexagonal systems, has also occurred in $h$-BaT$\mathrm{iO}_{3}$ by the classical reaction

$$
\begin{aligned}
& \mathbf{b} \rightarrow \mathbf{b}^{\prime}+\mathbf{b}^{\prime \prime} \\
& 1 / 3[\overline{1} 2 \overline{1} 0] \rightarrow 1 / 3[\overline{1} 100]+1 / 3[01 \overline{1} 0]
\end{aligned}
$$

Some of the perfect dislocations not lying in the basal plane had climbed. ${ }^{8}$ The Shockley half-partials were found to lie within widely extended planar stacking faults (EPSF). The fault vector of the portion of the stacking fault bordered by partial dislocations $D_{4}$ and $D_{5}$ in Eibl et al. ${ }^{8}$ was $\mathbf{R}_{F}=1 / 3[1 \overline{1} 01]$ while that of the other portion was $\mathbf{R}_{F}=1 / 3[\overline{1} 101]$, such that the fault vectors have opposite components in the basal plane: $[1 \overline{1} 00]$ and $[1100]$. It was concluded ${ }^{8}$ that the two fault vectors differed only in the components parallel to the basal plane, i.e. the prismatic components $1 / 3\langle 10 \overline{1} 0\rangle$ of the general form: $\mathbf{R}_{F}=(n / 6)$ $[0001]+1 / 3\langle 1010\rangle,{ }^{5}$ by vectors other than the lattice vectors. In addition, the two segments of stacking fault, separated by one of the Shockley half-partials, lie in two distinct basal planes (i.e., $(000 l)$ where $l=1-6)$ separated by $(1 / 2) \mathbf{c}$ where $\mathbf{c}=[0001]$.

In this study, the planar faults in hot-pressed $h-\mathrm{BaTiO}_{3}$ found to be embedded with partial dislocations were determined for their type, fault vectors, ${ }^{5}$ and the intermixed dislocations for Burgers vectors by the transmission electron microscopy (TEM). All dislocations were half-partials with $\mathbf{b}=1 / 3\langle\overline{1} 010\rangle$, consistent with those reported before by Eibl et al. ${ }^{8}$ Relationships between Burgers vectors and fault characteristics are discussed.

\section{Experimental Procedure}

A commercial hydrothermal $\mathrm{BaTiO}_{3} \operatorname{powder}^{5}$ (K-Plus ${ }^{\mathrm{TM}}$ ) of an average particle size of $\sim 120 \mathrm{~nm}$ purchased from Cabot ${ }^{B}$ (Boyertown, PA) was used in this study. The powder was stoichiometric and contained major impurities of residual carbon 
$(<1000 \mathrm{ppm})$, soluble chloride $(<250 \mathrm{ppm})$, and strontium $(<$ $500 \mathrm{ppm})$, according to the manufacturer. Samples were hot pressed at $1300^{\circ} \mathrm{C}$ for $2 \mathrm{~h}$ by a uniaxial pressure of $\sim 25.8 \mathrm{MPa}$ in a graphite die assembly using a Fujidempa (Osaka, Japan) multipurpose furnace equipped with a graphite resistance-heating element whose temperature was monitored by a W-Re thermocouple and a two-color pyrometer. Hot-pressed samples, while being kept in the graphite die, were furnace cooled to room temperature.

As-hot-pressed disks of $15 \mathrm{~mm}$ diameter were lightly polished to remove graphite and boron nitride (BN) coating from the graphite spacers that were inserted between the graphite punch and $\mathrm{BaTiO}_{3}$ samples before hot pressing. Thin foils for TEM were prepared by the conventional technique of cutting with a diamond-embedded saw, grinding, and polishing to an $\sim 30 \mu \mathrm{m}$ thickness, and $1 \mu \mathrm{m}$ of surface roughness before dimple grinding to an $\sim 10 \mu \mathrm{m}$ thickness in the center region. The thin sections were then $\mathrm{Ar}^{+}$ion-beam thinned (DuoMill ${ }^{\mathrm{TM}}$, Gatan, Pleasanton, CA) to electron transparency. Observations were made in a JEOL $^{\text {TM }}$ AEM3010 (Tokyo, Japan), with a double-tilting stage of $\pm 45^{\circ}$ for the $x$-tilt and $\pm 30^{\circ}$ for the $y$-tilt, operating at $300 \mathrm{kV}$.

\section{Results}

Stacking faults were observed in almost all $h$ - $\mathrm{BaTiO}_{3}$ grains. Grains were spherulitic in shape although not grown abnormally. ${ }^{2,9}$

\section{(1) General Observations-Fringe Patterns}

Selecter area diffraction patterns were indexed ${ }^{5}$ corresponding to the standard triangles of the stereographic projection. Seven sets of faults are discernible from one of the $h$ - $\mathrm{BaTiO}_{3}$ grains imaged in strong beam bright field (BF) (Fig. 1). Spot streaking suggests that the foil contains planar defects inclining to the beam direction at deviation parameter $s_{\mathbf{g}} \neq 0$ (Figs. 1(a) and (b)). ${ }^{5,10}$ Only six of them, designated as $F_{1}-F_{6}$, have been fully characterized. Faults $F_{5}$ and $F_{6}$ were visible (Fig. 1(a)) when imaged with $\mathbf{g}=\overline{1} 01 \overline{4}$. Faults $F_{5}$ and $F_{6}$ are partly superimposed in projection, producing a complex fringe pattern that remains symmetrical in BF. They are entirely similar to faults $F_{\mathrm{B}}$ and $F_{\mathrm{C}}$ in a previous report. ${ }^{5}$

Four dislocations, $d_{1}-d_{4}$, were detected in the field of view (Fig. 1(a)). Two of them, $d_{1}$ and $d_{2}$, with the respective Burgers vectors $\mathbf{b}_{d_{1}}$ and $\mathbf{b}_{d_{2}}$, lie within fault $F_{1}$, thus dividing $F_{1}$ into three segments, $F_{1}-F_{1}-F_{1}$, which show alternate fringe contrast. Using $\mathbf{g}=\overline{1014}$, the inner segment $F_{1}$ was visible (indicated by (O)) while the rest of the fault, on both sides of $F_{1}^{\prime}$, was invisible (indicated by $(\mathrm{X})$ ), i.e., fault contrast was reversed across both dislocations $d_{1}$ and $d_{2}$. Nevertheless, the fringe patterns revealed by a strong-beam BF image were symmetrical and complementary (for $s_{\mathrm{g}}=0$ ) about a foil center. ${ }^{11}$ Extreme fringe contrast was both dark in BF image, and the dark-dark (D-D) fringe pattern of $F^{\prime}{ }_{1}$ is indicated in Fig. 1(a). The fringes were however asymmetrical in CDF image where the extreme fringe contrast became dark-bright (D-B). The contrast characteristics are similar to those reported ${ }^{5}$ before, which allows us to conclude as of the $\alpha$-type fault ${ }^{11}$ with fault vectors $\left(\mathbf{R}_{F}\right)$, thus directly related to the lattice displacement vectors of hexagonal crystals.

Fault $F_{7}$ is bordered by dislocation $d_{3}$, as indicated in Fig. 1(c). Dislocation $d_{4}$ with Burgers vector $\mathbf{b}_{d_{4}}$ sits in the foil alongside with fault $F_{3}$. Causing no change in the fringe pattern, it is not in contact with the fault. Nevertheless, its presence indicates that $h$ - $\mathrm{BaTiO}_{3}$ was plastically deformed during hot pressing by the movement of dislocations $\mathbf{b}_{d_{4}}=1 / 3[10 \overline{10}]$.

Faults $F_{1}-F_{4}$ are invisible (X) while $F_{5}$ and $F_{6}$ remain visible (O) both from $\mathbf{g}=\overline{1} 01 \overline{4}$ and $1 \overline{1} 0 \overline{4}$. All these faults are invisible from $\mathbf{g}=\overline{2} 110$ and visible from $\mathbf{g}=2 \overline{1} \overline{1} 4$, accordingly (Figs. 1(a)-(d)). They are of a wedge shape as the foil thickness continuously decreases toward the left of the image.

\section{(2) Determination of Fault Vectors for Faults $\boldsymbol{F}_{1}-\boldsymbol{F}_{6}$ and $\boldsymbol{F}_{a}$} The eligible fault vectors for $F_{1}-F_{6}$ were determined by the three-step procedure ${ }^{5}$ described previously: (1) application of the invisibility criteria under diffraction contrast and under
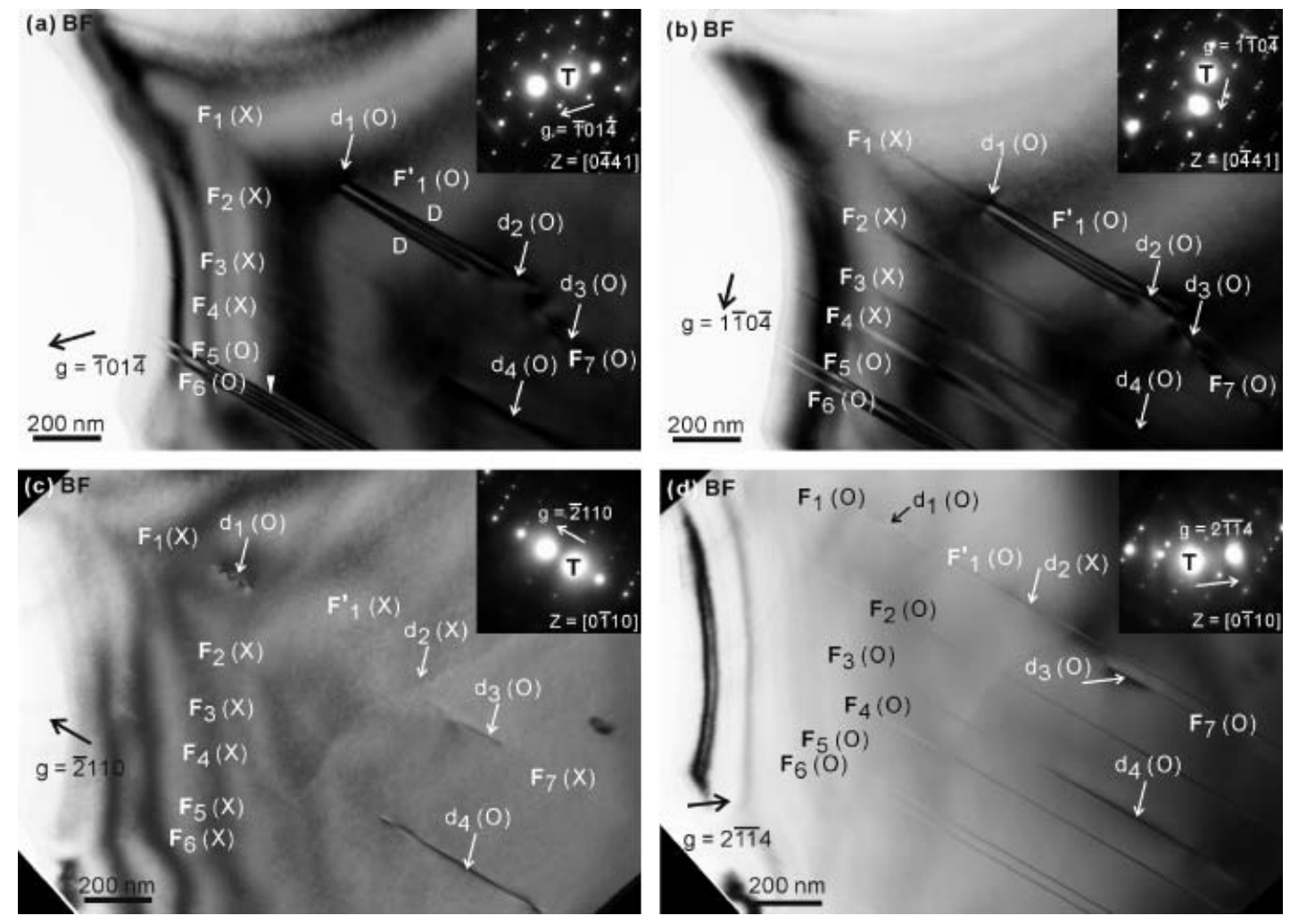

Fig. 1. Contrast analysis for faults $F_{1}-F_{6}$ using (a) $\mathbf{g}=\overline{1} 01 \overline{4}$, (b) $\mathbf{g}=1 \overline{1} 0 \overline{4}$, (c) $\mathbf{g}=\overline{2} 110$, and (d) $\mathbf{g}=2 \overline{1} \overline{1} 4$ (bright-field image, transmission electron microscopy). 
high-resolution imaging; (2) count of the extra $c$-layers; and (3) identification of displacement along prismatic directions, were followed to determine the eligible fault vectors for $F_{1}-F_{6}$.

(A) Faults $F_{1}-F_{4}$ : Faults $F_{1}-F_{4}$ are visible under $\mathbf{g}=2 \overline{1} \overline{1} 4$, (Fig. $1(\mathrm{~d})), 10 \overline{1} 3$, and $1 \overline{1} 0 \overline{3}$ while $F_{5}$ and $F_{6}$ are visible under $\mathbf{g}=\overline{1} 01 \overline{4}$ and $1 \overline{1} 0 \overline{4}$ in $Z=[0 \overline{4} 41]$ (Figs. 1(a) and (b)).The results for fault vectors $\mathbf{R}_{F_{1}}, \mathbf{R}_{F_{2}}, \mathbf{R}_{F_{3}}$, and $\mathbf{R}_{F_{4}}$ are shown in Table I; the faults exhibit five, six, and eight layers. All the faults determined here are of the $\mathbf{R}_{F_{(I)}}$ type, none of the $\mathbf{R}_{F_{(\mathrm{I})}} \mathbf{R}_{F(\mathrm{I})}$ type, for example $F_{\mathrm{A}}$ in $\mathrm{Wu}$ and $\mathrm{Lu},{ }^{5}$ has been observed.

The fault vectors for $\mathbf{R}_{F_{1}}$ are $1 / 6[02 \overline{2} 1], 1 / 6[\overline{2} 021]$, and $1 / 6[2 \overline{2} 01]$, consistent with observed diffraction contrast and a five-layer fault (with the stacking sequence of $(\boldsymbol{c h c}) \boldsymbol{c}(\mathbf{c h} c)$, containing one extra $c$-layer) determined from the HR image. The characteristic zig-zag feature of the $(\boldsymbol{c h c})(\mathbf{c h} c)$ stacking sequence (with the $c$-layer indicated by open circles and the $h$-layer by full circles) in unfaulted $h$ - $\mathrm{BaTiO}_{3}$ was identified from $Z=[1210]$. Both the zig-zag occurred at the $h$-layer (i.e., the $B$-layer in $(\mathrm{CBC})(\mathrm{ABA})$ ) and is indicated by full circles in Fig. 2(a). Fault $F_{1}$ may be described by a total of nine layers (i.e., R5+L4) consisting of five right-inclined zig layers (designated R5) and four zag layers (designated L4) by counting twice the turning point at the $h$-layers (indicated by full circles). Tilting the foil by $30^{\circ}$ clockwise to the consecutive $[0 \overline{1} 10]$, fault $F_{1}$ containing five layers in total, although embedded in the unfaulted $(c \mathbf{h c})(\mathbf{c h} c)$ stacking sequence of $h-\mathrm{BaTiO}_{3}$, could still be differentiated by the three $c$-layers appearing between two $h$-layers, as indicated by open circles (for $c$-layers) and full circles (for $h$-layers) in Fig. 2(b). This is shown in Fig. 2(b) from the framed region of Fig. 2(b) at a higher magnification: the lattice spacings of $d_{2 \overline{1} \overline{0} 0}=0.286 \mathrm{~nm}$ and $d_{0006}=0.233 \mathrm{~nm}$ are also indicated. Unlike the projection on $(1 \overline{2} 10)$, whether the $h$-layer or the $c$-layer has shifted $^{5}$ along [2110] cannot be discerned from $Z=[0 \overline{1} 10]$, although the fault-containing five layers may still be differentiated.

On the other hand, $\mathbf{R}_{F_{2}}$ and $\mathbf{R}_{F_{3}}$ are six-layer faults with (chc)cc(chc), whereas $\mathbf{R}_{F_{4}}$ is eight-layered with (chc) $\operatorname{cccc}(\mathbf{c h} c)$. The eligible fault vectors for $F_{1}-F_{4}$ are summarized in the "number of layers" column in Table I.

(B) Faults $F_{1}^{\prime}, F_{5}$, and $F_{6}$ : Faults $F_{1}^{\prime}, F_{5}$, and $F_{6}$ exhibit a (chc) $\boldsymbol{c}(\mathbf{c h} c)$ stacking sequence, identical to that of $F_{1}$. Eligible fault vectors could only be confirmed by investigating HR images for $Z=[1 \overline{2} 10]$ where five-layer faults containing one extra $c$-layer may be distinguished. For faults $F^{\prime}{ }_{1}, F_{5}$, and $F_{6}$, only three of the six $\langle 0110\rangle$ prismatic directions $\mathbf{R}_{F_{5}}=\mathbf{R}_{F_{6}}=\mathbf{R}_{F_{1}^{\prime}}=$ $1 / 6[0 \overline{2} 21], 1 / 6[20 \overline{2} 1]$, and $1 / 6[\overline{2} 201]$ are valid, referring to the "number of layers" column in Table I. The eligible fault vectors (Table I), projected on (0001), are illustrated schematically in Fig. 3.

There is a $60^{\circ}$-rotation correspondence between fault vectors $\mathbf{R}_{F^{\prime}}=1 / 6[20 \overline{2} 1]$ and $\mathbf{R}_{F_{1}}=1 / 6[02 \overline{2} 1]$ (Table I), and faults $F_{1}$ and $F^{\prime}{ }_{1}$ exhibit contrast reversal across dislocations $d_{1}$ and $d_{2}$ (demonstrated in Figs. 1(a) and (b)). The fact that a clockwise $60^{\circ}$ rotation of $F_{1}$ (and likewise for faults $F_{2}-F_{6}$ ) about [0001]
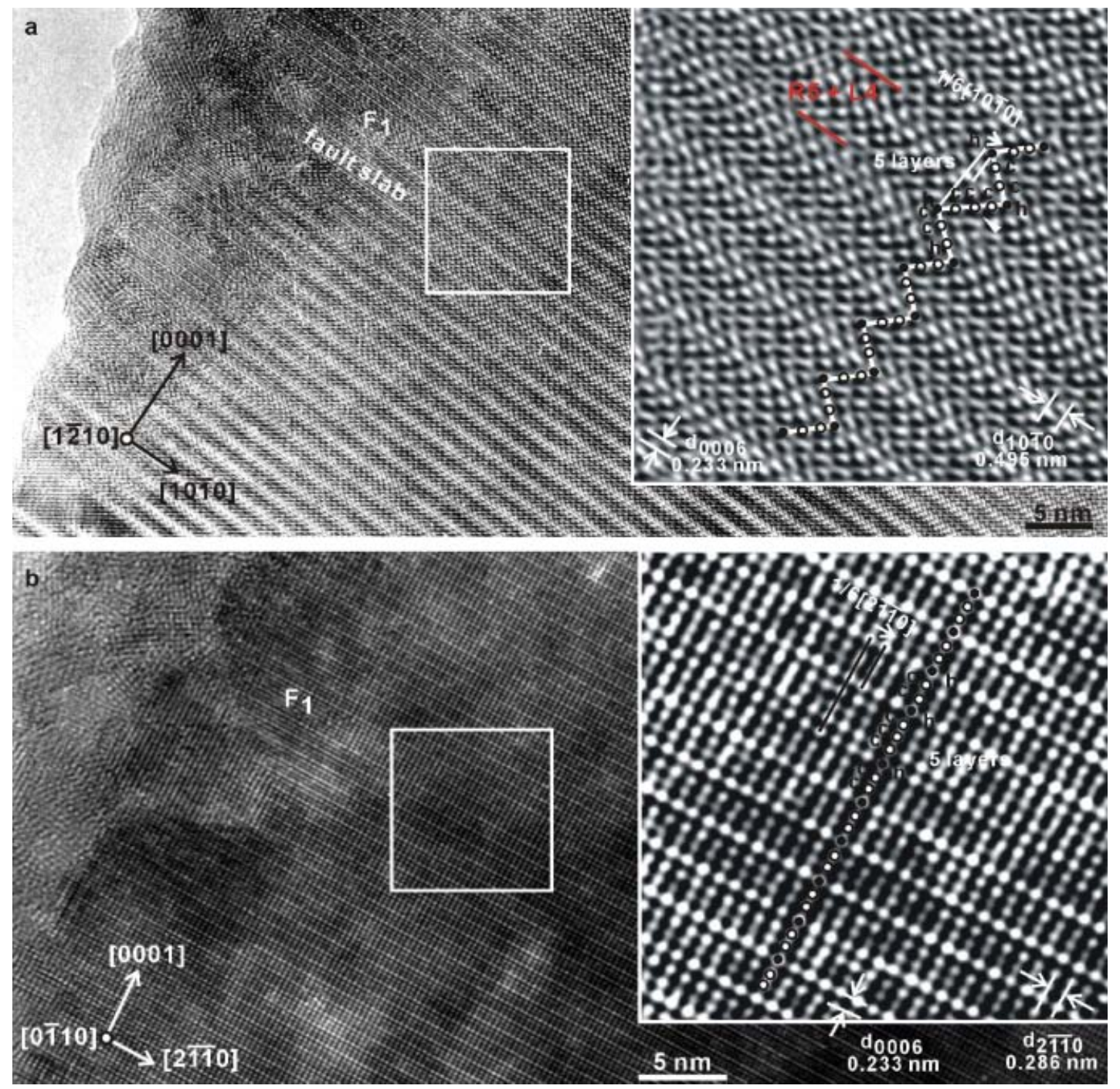

Fig. 2. High-resolution images of fault $F_{1}, \mathbf{R}_{F_{1}}=1 / 6[02 \overline{2} 1]$; a five-layer fault containing one extra $c$-layer viewed from (a) $Z=[1 \overline{2} 10]$, and (b) [0110] (transmission electron microscopy). 
Table I. Fault Vectors for $F_{1}, F_{1}^{\prime}, F_{2}, F_{3}, F_{4}, F_{5}, F_{6}, F_{a}$, and $F^{\prime}{ }_{a}$ Determined by the $2 \pi \mathrm{g} \cdot \mathbf{R}=\mathbf{0}$ or $2 n \pi$ Invisibility Criteria $\left(\mathbf{R}_{F}\right.$ of 12 Variations), High-Resolution Imaging (HR), and Number of Layers (of Three Eligible Vectors)

\begin{tabular}{|c|c|c|c|c|}
\hline Type & Fault & HR (layers) & $\mathrm{R}_{F}$ & Number of layers \\
\hline \multirow{6}{*}{$\mathbf{R}_{F(I I)}$} & $F_{1}, F_{\mathrm{a}}$ & 5 & $1 / 6[02 \overline{2} 1], 1 / 6[\overline{2} 021], 1 / 6[2 \overline{2} 01] 5$ layers & $1 / 6[02 \overline{2} 1], 1 / 6[\overline{2} 021], 1 / 6[2 \overline{2} 01]$ \\
\hline & $F_{2}, F_{3}$ & 6 & $1 / 3[0111], 1 / 3[10 \overline{1} 1], 1 / 3[1101] 6$ layers & $1 / 3[0 \overline{1} 11], 1 / 3[10 \overline{1} 1], 1 / 3[1101]$ \\
\hline & $F_{4}$ & 8 & $1 / 6[0 \overline{2} 25], 1 / 6[20 \overline{2} 5], 1 / 6[\overline{2} 205] 9$ layers & $1 / 3[01 \overline{1} 2], 1 / 3[\overline{1} 012], 1 / 3[1 \overline{1} 02]$ \\
\hline & $F^{\prime}{ }_{1}$ & 5 & $1 / 6[0 \overline{2} 21], 1 / 6[20 \overline{2} 1], 1 / 6[\overline{2} 201] 5$ layers & $1 / 6[0 \overline{2} 21], 1 / 6[20 \overline{2} 1], 1 / 6[\overline{2} 201]$ \\
\hline & $F_{5}$ & & $1 / 3[0111], 1 / 3[1011], 1 / 3[1 \overline{1} 01] 6$ layers & \\
\hline & $F_{a}^{\prime}$ & & $1 / 6[02 \overline{2} 5], 1 / 6[\overline{2} 025], 1 / 6[2205] 9$ layers & \\
\hline
\end{tabular}

produces non-eligible shear vectors suggests that they are not the $\pi$-rotation type. ${ }^{12,13}$

(c) Fault $F_{a}$ and Partial Dislocation $d_{a}$ : The fault shown in Fig. 4(a) found in other areas of the foil is interrupted by one of two partials $d_{a}$ with Burgers vector $\mathbf{b}_{d_{a}}=1 / 3[1010]$ to two segments $F_{a}$ with fault vectors 1/6[0221],1/6[2021], and $1 / 6[2 \overline{2} 01]$, and $F^{\prime}{ }_{a}$ with fault vectors $1 / 6[0 \overline{2} 21], 1 / 6[20 \overline{2} 1]$, and $1 / 6[2201]$ when they differ by a $60^{\circ}$ rotation about [0001] (Table I). The true line direction $\mathbf{u}_{\mathrm{t}}=[\overline{2} 110]$ of partial dislocation $d_{a}$ determined by trace analysis suggests that it is a $30^{\circ}$ mixed type. Fault slab, as indicated, can be differentiated unambiguously in Figs. 4(b) and (c). Both segments being fivelayer faults of the stacking sequence $(\boldsymbol{c h c}) \boldsymbol{c}(\mathbf{c h} c)$ containing one extra $c$-layer are evidenced from $\mathrm{HR}$ images on either side of partial $d_{a}$ shown in Fig. 4(d) for $F_{a}$ and Fig. 4(e) for $F^{\prime}{ }_{a}$. The nine-layer stacking sequence of right-inclined fault $F_{a}$ containing (R5+L4)-layers (indicated in Fig. 4(d)) remains unaltered on moving across partial dislocation $d_{a}$ to left-inclined fault $F^{\prime}{ }_{a}$ of (L5+R4)-layers (Fig. 4(e)). Moving across the partial dislocation, similar to faults $F_{2}-F_{6}$, the fault vectors have simply rotated by $60^{\circ}$. Similar to $F_{1}$, the stacking sequence is retained across the partial, i.e. $d_{1}$ and $d_{2}$ in $F_{1}$ and $d_{a}$ in $F_{a}$ (Table II).

\section{(3) Determination of Burgers Vectors, Line Directions for Dislocations Associated with Faults}

All dislocations having Burgers vectors $\mathbf{b}=1 / 3\langle 1 \overline{1} 00\rangle$ are prism-plane partials. The term "prism-plane dislocations" sug-

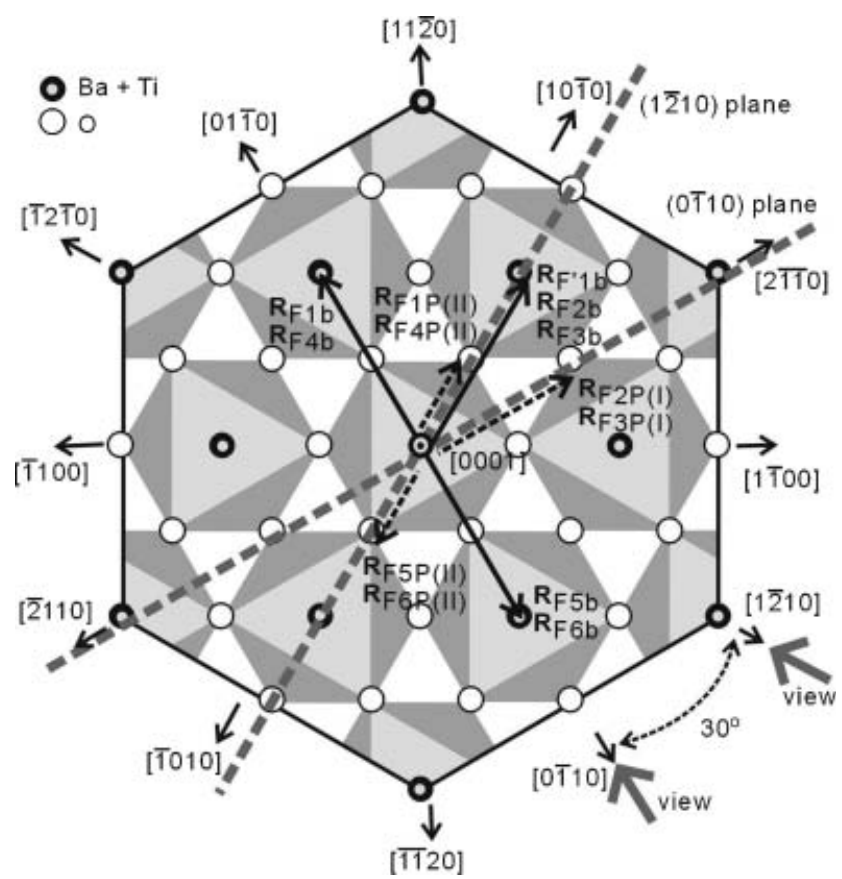

Fig. 3. Fault vectors projected on (0001) showing the prismatic components, noting that $F_{5}, F_{6}$, and $F_{2}, F_{3}$ although sharing the same prismatic component $\langle 01 \overline{1} 0\rangle$, were $120^{\circ}$ between each other. gested by Heuer ${ }^{14}$ is adopted here instead of prismatic dislocations to avoid confusion with prismatic loops. Burgers vectors are as follows: $\mathbf{b}_{d_{1}}=1 / 3[1 \overline{1} 00], \mathbf{b}_{d_{2}}=1 / 3[0 \overline{1} 10]$, and $\mathbf{b}_{d_{3}}=$ $\mathbf{b}_{d_{4}}=1 / 3[10 \overline{1} 0]$, as given in Table II. True directions have been determined by trace analysis. Only dislocation $d_{3}$ is of the screw type; others are of mixed type of $60^{\circ}$ for $d_{1}$ and $d_{4}$, and $30^{\circ}$ for $d_{2}$. The prism-plane partials are glissile in the (0002) basal plane in which they are lying.

\section{Discussion}

We have compared the present results of fault vectors with those reported ${ }^{5}$ previously, placing emphasis particularly on why dislocations $d_{1}$ and $d_{2}$ produce a fault fringe contrast reversal.

\section{(1) Eligible Fault Vectors}

Fault vectors determined in this study belong to one of the two general types, ${ }^{5} \mathbf{R}_{F_{(\mathrm{I})}}$ and $\mathbf{R}_{F_{(\mathrm{II})}}$, of the stacking faults in $h$-BaT$\mathrm{iO}_{3}$. They correspond to $\mathbf{R}_{F_{(\mathrm{II})}}=n / 6[0001]+1 / 3\langle 10 \overline{1} 0\rangle, n=1$, 2,4 , and 5 of the extrinsic nature. Each fault shows three possible fault vectors (Table I) as determined accordingly.

Interrupting the $h-\mathrm{BaTiO}_{3}$ stacking sequence by inserting extra $c$-layer(s), contributing to the basal component $(n / 6) \mathbf{c}$ of fault vectors $\mathbf{R}_{F}{ }^{5}$ has been clearly evidenced from $\mathrm{HR}$ images, for example (1/6)c in Fig. 2(a) for fault $F_{1}$. Displacements of the $(c h c)(c h c)$ zig-zag feature ${ }^{3,5,9}$ along $\langle 10 \overline{1} 0\rangle$, for example $1 / 6[10 \overline{10}]$ in Fig. 2(a), have also enabled to determine the prismatic component $\mathbf{p}_{(\mathrm{II})}^{\prime}=1 / 3\langle 10 \overline{1} 0\rangle$. The unfaulted stacking sequence modified by extra $c$-layers inserted along [0001] taken ${ }^{5}$ to justify that only three prismatic components of the shear vectors along $\langle 10 \overline{1} 0\rangle$ are valid fault vectors is equally applicable to faults analyzed here.

When examining the stacking sequence along [0001], possible shear vectors may again be grouped into two major classes depending on where in the unfaulted stacking sequence the first extra $c$-layer is inserted. The insertion of the extra $c$-layer occurring at immediately after (CBC) generates left-inclined faults, e.g., $F_{a}^{\prime}$ in Fig. 4(e) (as shown in Fig. 5(a)). ${ }^{5}$ Right-inclined faults, e.g., $F_{1}$ in Fig. 2(a) and $F_{a}$ in Fig. 4(d), result from insertion after (ABA) (Fig. 5(b)) ${ }^{5}$

All faults identified in the hot-pressed $h-\mathrm{BaTiO}_{3}$ ceramics, i.e. $F_{\mathrm{A}}-F_{\mathrm{D}}$ in $\mathrm{Wu}$ and $\mathrm{Lu},{ }^{5}$ and $F_{1}-F_{6}$ in the present work are included in the stacking sequences for the five-, six-, seven-, eight-, and nine-layer faults shown ${ }^{5}$ schematically for the two groups of faults. So far, not all predicted faults (shown in Figs. 5(a) and (b)) have been encountered.

\section{(2) Dislocation Glide or Climb in Basal Planes}

The difference of $1 / 3[1 \overline{1} 00]$ between fault vectors $\mathbf{R}_{F_{1}}=$ $1 / 6[02 \overline{2} 1]$ and $\mathbf{R}_{F^{\prime}}=1 / 6[2021]$ is equal to the Burgers vector of partial dislocation $d_{1}$, and that between $\mathbf{R}_{F^{\prime}}$ and $\mathbf{R}_{F_{1}}$ is again equal to $\mathbf{b}_{d_{2}}=1 / 3[0 \overline{1} 10]$ of partial dislocation $d_{2}$. As both faults contained one extra $c$-layer (as evidenced by a similar five-layered fault $F_{a}$ shown in Fig. 4(d)), dislocations $d_{1}$ and $d_{2}$ have 



Fig. 4. Fault $F_{a}$ interrupted by partial dislocation $d_{a}$, (a) bright-field image showing two segments $F_{a}$ and $F_{a}{ }_{a}$; (b) and (c) are high-resolution images taken from either side of partial $d_{a}$; (d) and (e) are higher magnifications of the local regions of (b) and (c).

moved in the (0002) fault plane by glide, leaving the c-component of the fault vectors unchanged.

The unit lattice translation vectors are possible Burgers vectors for perfect dislocations. We have in $h-\mathrm{BaTiO}_{3}$ the basal dislocation, $\mathbf{b}_{\mathrm{B}}=1 / 3\langle 11 \overline{2} 0\rangle$, the prism-plane dislocation, $\mathbf{b}_{\mathrm{Pr}}=$ $\langle 10 \overline{1} 0\rangle$, and the pyramidal dislocation, $\mathbf{b}_{\mathrm{Py}}=1 / 3\langle 20 \overline{2} 1\rangle$. Similar to hcp A3 metals, $\langle 11 \overline{2} 0\rangle$ are the close-packed directions in $h$ $\mathrm{BaTiO}_{3}$. Basal slip along the close-packed directions, $1 / 3\langle 11 \overline{2} 0\rangle$ (0001), is the easiest slip system. However, unlike the configurations reported by Eibl et al. ${ }^{8}$ who found basal dislocations $\mathbf{b}_{\mathrm{B}}$ further dissociated into two prism-plane half-partials (by Eq.

Table II. True Directions, Type of Dislocations and the Relevant Slip Plane

\begin{tabular}{lcccl}
\hline Dislocation & True direction $\mathbf{u}_{t}$ & Burgers vector $\mathbf{b}$ & Slip plane & Dislocation type \\
\hline$d_{1}$ & {$[0 \overline{1} 10]$} & $1 / 3[1 \overline{1} 00]$ & $(0002)$ & Mixed $\left(60^{\circ}\right)$ \\
$d_{2}$ & {$[1 \overline{2} 10]$} & $1 / 3[0 \overline{1} 10]$ & $(0002)$ & Mixed $\left(30^{\circ}\right)$ \\
$d_{3}$ & {$[\overline{1} 010]$} & $1 / 3[10 \overline{1} 0]$ & $(0002)$ & Screw \\
$d_{4}$ & {$[\overline{1} 100]$} & $1 / 3[10 \overline{1} 0]$ & $(0002)$ & Mixed $\left(60^{\circ}\right)$ \\
$d_{a}$ & {$[\overline{2} 110]$} & $1 / 3[1010]$ & $(0002)$ & Mixed $\left(30^{\circ}\right)$ \\
\hline
\end{tabular}

(1)), only dissociated $\mathbf{b}_{\operatorname{Pr}}$ have been identified in hot-pressed samples, i.e., $d_{1}, d_{2}$ coplanar with fault $F_{1}$, and $d_{3}, d_{4}$ coplanar with $F_{7}$ and $F_{3}$, respectively. Perfect basal dislocations with $\mathbf{b}_{\mathrm{B}}=$ $1 / 3\langle 11 \overline{2} 0\rangle$ that would indicate possible shear in this direction have not yet been identified in hot-pressed $h-\mathrm{BaTiO}_{3}$.

However, Shockley half-partials, $d_{1}$ and $d_{2}$, originated from the dissociation of basal dislocations $\mathbf{b}_{\mathrm{B}}=1 / 3\langle 1120\rangle$ by the reaction $\mathbf{b}_{\mathrm{B}} \rightarrow \mathbf{b}_{d_{1}}+\mathbf{b}_{d_{2}}$ :

$$
1 / 3[1 \overline{2} 10] \rightarrow 1 / 3[1 \overline{1} 00]+1 / 3[0 \overline{1} 10]
$$

As basal dislocations had subsequently dissociated into two prism-plane half-partials (by Eq. (2)), they were observed in the present samples. Shear still occurs principally along $\langle 11 \overline{2} 0\rangle$ in $h$ - $\mathrm{BaTiO}_{3}$, only that such basal dislocations induced plastically had dissociated. Therefore, plastic deformation took place predominantly through the easiest slip system $1 / 3\langle 11 \overline{2} 0\rangle(0002)$ in $h-\mathrm{BaTiO}_{3}$ when a powder of $\sim 120 \mathrm{~nm}$ was hot pressed at $1300^{\circ} \mathrm{C}$.

The dissociation of basal dislocations into partials $d_{1}$ and $d_{2}$ with $\mathbf{b}_{d_{1}}$ and $\mathbf{b}_{d_{2}}$ takes place by glide in (0002), the B-layer in (CBC) stacking sequence, as the component of faults $F_{1}$ and $F_{1}$ is the same. The fault segment encompassed by partials $d_{1}$ 

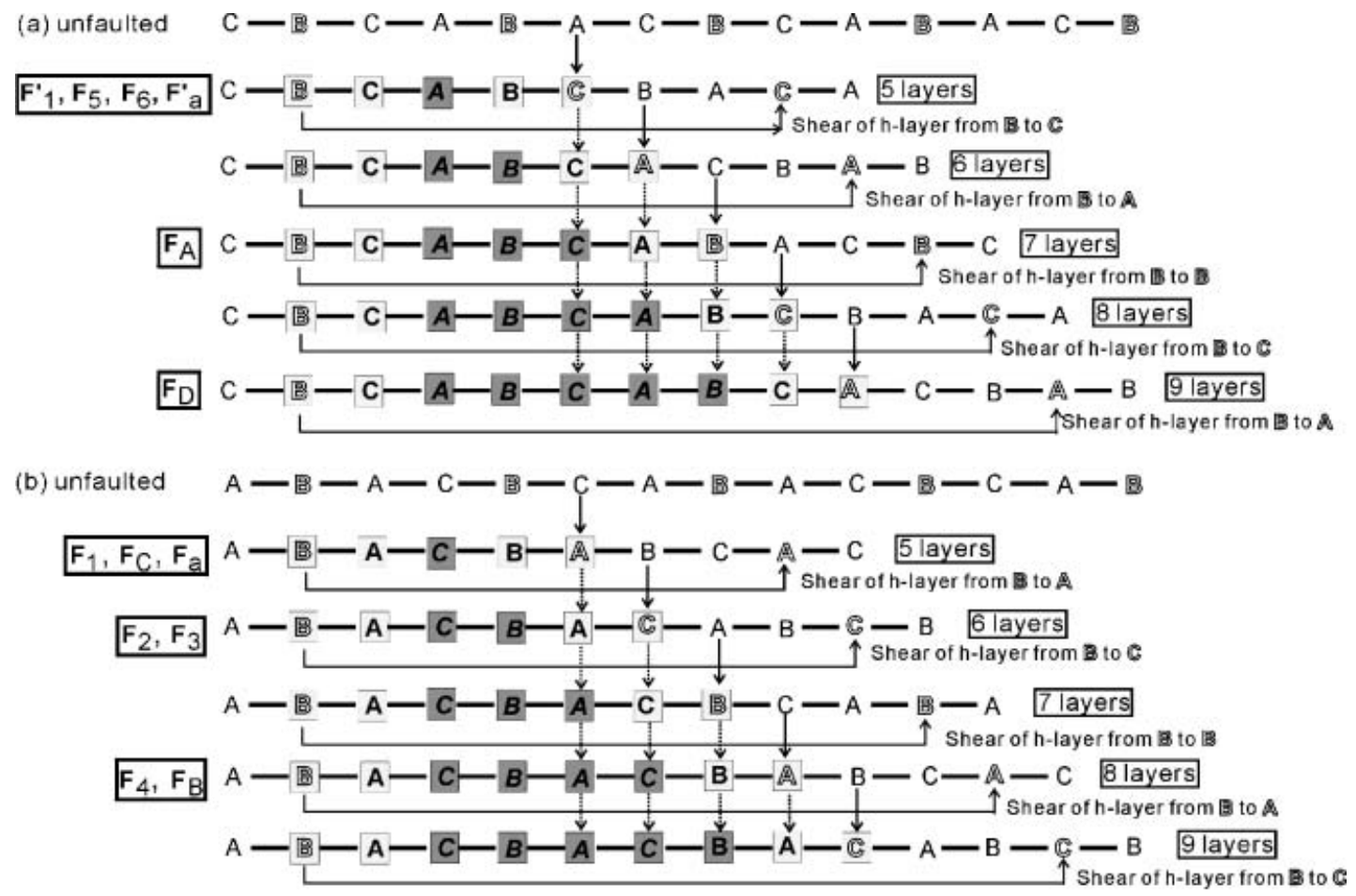

Fig. 5. Stacking sequence of the unfaulted $h$ - $\mathrm{BaTiO}_{3}$ and those of the five-, six-, seven-, eight-, and nine-layer faults with shear on (0002), including faults (a) inclined to the left, and (b) inclined to the right. ${ }^{5}$

and $d_{2}$ is therefore a complex extrinsic stacking fault (CESF). The complex fault enveloped by partials $d_{1}$ and $d_{2}$ is fault segment $F_{1}^{\prime}$ that contains one extra $c$-layer with fault vector $\mathbf{R}_{F_{1}^{\prime}}=$ $1 / 6[20 \overline{2} 1]$ (Fig. 2(a)). The fault vectors of $F_{1}$ and $F^{\prime}{ }_{1}$ belong to two crystallographically distinctive groups of $1 / 6\langle 02 \overline{2} 1\rangle$, whose projections on (0002) together with $\mathbf{b}_{d_{1}}$ and $\mathbf{b}_{d_{2}}$ are shown schematically in Fig. 6.

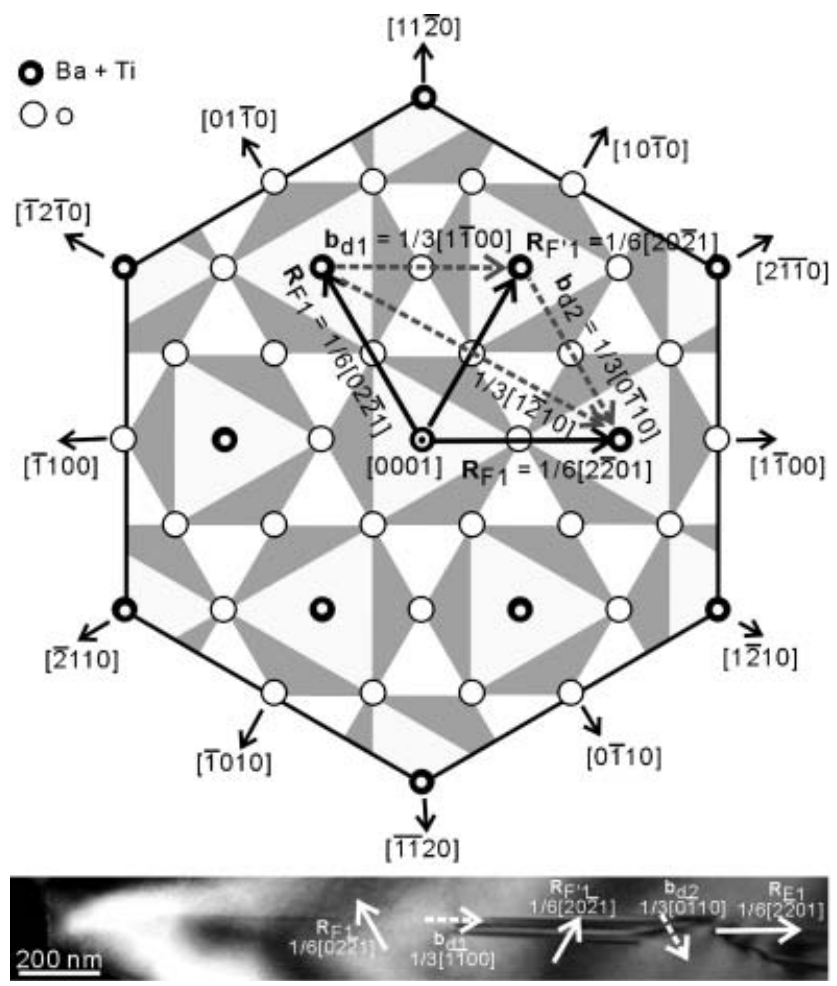

Fig. 6. Dissociation of basal dislocation with $\mathbf{b}_{\mathrm{B}}=1 / 3[1 \overline{2} 10]$ to prismplane partial dislocations $\left(\mathbf{b}_{\mathrm{Pr}}\right)$ with $\mathbf{b}_{d_{1}}=1 / 3[1 \overline{1} 00]$ and $\mathbf{b}_{d_{2}}=1 / 3[0 \overline{1} 10]$ in the (0002) plane.
Compared with the stacking fault energy $\gamma_{\mathrm{SF}} \approx 108 \mathrm{~mJ} / \mathrm{m}^{2}$ of $t$ - $\mathrm{BaTiO}_{3},{ }^{15}$ the larger separation $(d)$ between a pair of half-partials, $d_{1}$ and $d_{2}$, of $\sim 500 \mathrm{~nm}$ (Fig. 1(a)) indicates a significantly lower $\gamma_{\mathrm{SF}}$ in $h-\mathrm{BaTiO}_{3}$. However, this value cannot be estimated at present as no shear modulus for $h-\mathrm{BaTiO}_{3}$ is available in the literature. Partials separated by $d \approx 120 \mathrm{~nm}$ found by Eibl et al., ${ }^{8}$ being much narrower, may not lie in the same plane but in planes differing by $(1 / 2) \boldsymbol{c}$ indicate a climb dissociation of $\mathbf{b}_{\mathrm{B}}$ into half-partials (nevertheless, similarly, by Eq. (2)). Fault segments on both sides of the partials $D_{4}$ and/or $D_{5}$ in Eibl et al. would have distinctive fault vectors with a characteristic stacking sequence containing different extra $c$-layers. This necessitates climb of partial dislocations upon dissociation in prism planes at the interface of the two stacking faults. One of a pair of halfpartials migrated by a mixed glide in (0002) and climb in (1010) has indeed been determined. ${ }^{16}$

The glide of $d_{1}$ with $\mathbf{b}_{d_{1}}=1 / 3[1 \overline{1} 00]$ on (0002), the fault plane of $F_{1}$, has effectively rotated $\mathbf{R}_{F_{1}}=1 / 6[02 \overline{2} 1]$ by $60^{\circ}$ clockwise to $\mathbf{R}_{F_{1}^{\prime}}=1 / 6[20 \overline{2} 1]$. A complex stacking fault is created extrinsically in fault segment $F^{\prime}{ }_{1}$ with $\mathbf{R}_{F^{\prime}}$. Partial dislocation $d_{2}$ with $\mathbf{b}_{d_{2}}=1 / 3[0 \overline{1} 10]$ then restores $\mathbf{R}_{F^{\prime}}{ }_{1}$ back to $\mathbf{R}_{F_{1}}$ in a similar fashion. Thus, bordering between faults $F_{1}$ and $F^{\prime}{ }_{1}$ is an interface characterized by half-partial $\mathbf{b}_{d_{1}}=1 / 3[1 \overline{1} 00]$ (Fig. 6). Analogously, the interface across $F^{\prime}{ }_{1}$ and $F_{1}$ is generated by $\mathbf{b}_{d_{2}}=1 / 3[0 \overline{1} 10]$. Consequently, the configuration of $F_{1}$ is resumed and $\mathbf{R}_{F_{1}}$ restored after the trailing half-partial, $\mathbf{b}_{d_{2}}$. Fault segments $F_{1}-F_{1}{ }_{1}-F_{1}$ separated by dislocations $d_{1}$ and $d_{2}$ did not have identical shear vectors (Table I) due to the displacement of $\mathbb{B} \rightarrow \mathbb{A}$ (for $F_{1}$ ) versus $\mathbb{B} \rightarrow \mathbb{C}$ (for $F^{\prime}{ }_{1}$ ) in (0002) (Fig. 5). ${ }^{5} \mathrm{Nev}-$ ertheless, the total number of nine layers in $F_{1}$ and $F^{\prime}{ }_{1}$ remains unchanged across half-partials $d_{1}$ and $d_{2}$, i.e., (R5+L4) to (L5+R4), from a right-inclined fault $\left(F_{1}\right.$ in Fig. 2(a)) to a leftinclined one (similar to that of $F^{\prime}{ }_{a}$ ) in Fig. 4(e)). Dissociation of perfect basal dislocation $\mathbf{b}_{\mathrm{B}}$ by a pure glide in (0002) has resulted in an extended separation between half-partials, such as that observed for $F_{1}-F_{1}^{\prime}$ at $d \approx 500 \mathrm{~nm}$ (Fig. 1(a)). Indeed, a separation of $d>320 \mathrm{~nm}$ for $d_{a}$ and its pair partial (which should have been located left of $d_{a}$ (Fig. 4(c)) if not milled off) was estimated from a lower magnification strong-beam image. When dissociation occurs by climb, such as that found by Eibl et $a .^{8}$ or a 
mixed mechanism, ${ }^{16}$ half-partials would have separated the initial fault into two fault segments consisting of different extra $c$ layers, i.e. differing in the component of fault vectors $\mathbf{R}_{F_{(I I)}}$. Planar faults in $h$ - $\mathrm{BaTiO}_{3}$ intersected by glide-dissociated halfpartials and divided into two or more segments of dissimilar fault vectors (Table I) but of similar stacking sequence $(\boldsymbol{c h c}) \boldsymbol{c}(\mathbf{c h} c)$ are found with $F_{a}$ and $F_{a}^{\prime}$ (Figs. 4(d)-(e)). Whether climb dissociation ${ }^{8}$ of $\mathbf{b}_{\mathrm{B}}$ generated by plastic deformation occurs at all may be investigated by determining the fault vector for each of the fault segments. Climb of partials must occur whenever faults with different extra $c$-layers are encountered in dislocation dissociation. Fault vectors of such segments suggest that the intersecting dislocations have climbed by $(n / 6)[0001]$ from one segment to the next, for example 1/2[0001] reported by Eibl et al. ${ }^{8}$ and $\mathrm{Wu}$ et al. ${ }^{16}$ If there are dislocations intersecting between a five-layer fault, e.g. $F_{1}$ and a six-layer fault, e.g. $F_{2}$, climb of $\mathbf{b}=1 / 3[1 \overline{1} 00]$ by $1 / 6[0001]$ must occur upon crossing from $\mathbf{R}_{F_{1}}=1 / 6[02 \overline{2} 1]$ to $\mathbf{R}_{\mathrm{F}_{2}}=1 / 3[10 \overline{1} 1]$, although such dislocations were not observed here. Other combinations with different extra $c$-layers in stacking faults are therefore possible. This has consequently produced discrepant separations between half-partials, such as $d \approx 500 \mathrm{~nm}$ determined in the present study and $\sim 120 \mathrm{~nm}$ reported by Eibl et al. ${ }^{8}$ and $\sim 47-195 \mathrm{~nm}$ by Wu et al. ${ }^{16}$

\section{Conclusions}

Eligible fault vectors determined for faults in metastably retained $h$-BaTiO ${ }^{3}$ with dislocations embedded were similar to those without the intersecting dislocations. Only three of the six prismatic components of $\langle 10 \overline{1} 0\rangle$ were eligible for type II faults $F_{1}-F_{6}$, and $F_{a}$ (with $\mathbf{R}_{F_{(I I)}}$ fault vectors) analyzed here.

During hot pressing, the Shockley half-partials with $\mathbf{b}_{\mathrm{Pr}}=$ $1 / 3\langle 0 \overline{1} 10\rangle$ were dissociated from basal dislocations with $\mathbf{b}_{\mathrm{B}}=$ $1 / 3\langle 11 \overline{2} 0\rangle$ by glide in (0002) and/or climb in prism planes of $h$ $\mathrm{BaTiO}_{3}$. The interface dislocation with $\mathbf{b}_{\mathrm{Pr}}=1 / 3\langle 0110\rangle$ has effected a $60^{\circ}$ rotation of fault segment $F_{1}$ with $\mathbf{R}_{F_{1}}$ to $F^{\prime}{ }_{1}$ with $\mathbf{R}_{F^{\prime}{ }_{1}}$ about [0001], and then from $F^{\prime}{ }_{1}$ back to $F_{1}$ by another rotation of $60^{\circ}$ successively. Fault segments $F_{1}-F_{1}$ and $F_{1}{ }_{1}-F_{1}$ are bordered by a pair of half-partials, $d_{1}$ and $d_{2}$, one leading and the other trailing. The fault segment $F^{\prime}{ }_{1}$ enveloped by half-partials is a CESF.

\section{References}

${ }^{1}$ K. W. Kirby and B. A. Weschler, "Phase Relations in the Barium-Titanium Oxide System," J. Am. Ceram. Soc., 74 [8] 841-7 (1991).

${ }^{2}$ M. H. Lin and H. Y. Lu, "Hexagonal Retention in MgO-Doped $\mathrm{BaTiO}_{3}$," Philos. Mag. A., 81 [1] 181-96 (2001).

${ }^{3}$ I. E. Grey, C. Li, L. M. D. Cranwick, R. S. Roth, and T. A. Vanderah, "Structure Analysis of the $6 \mathrm{H}-\mathrm{Ba}\left(\mathrm{Ti}, \mathrm{Fe}^{3+}, \mathrm{Fe}^{4+}\right) \mathrm{O}_{3-\delta}$ Solid Solution," J. Solid Stat. Chem., 135 [2] 312-21 (1998).

${ }^{4} \mathrm{H}$. Mostaghaci and R. J. Brook, "Kinetics of Hot-Pressing of $\mathrm{BaTiO}_{3}$ Ceramics,” Br. Ceram. Trans. J., 84, 963-73 (1985).

${ }^{5}$ Y. C. Wu and H. Y. Lu, "Planar Defects in Metastably Retained Hexagonal $\mathrm{BaTiO}_{3}$ Ceramics: $\alpha$-Type Extended Stacking Faults," Philos. Mag., 84 [32] $3467-$ 81 (2004).

${ }^{6}$ E. Sawaguchi, Y. Akshihe, and M. Kobayashi, "Ferroelectric Behaviour in Hexagonal Type Barium Titanate," Jpn. J. Appl. Phys., 24 [Suppl. 24-2] 252-4 (1985).

${ }^{7}$ A. Feteria, K. Sarma, N. M. Alfore, I. M. Reaney, and D. C. Sinclair, "Microwave Dielectric Properties of Gallium-Doped Hexagonal $\mathrm{BaTiO}_{3}$ Ceramics," J. Am. Ceram. Soc., 86 [3] 511-3 (2003).

${ }^{8}$ O. Eibl, P. Pongratz, and P. Skalicky, "Extended Defects in Hexagonal BaT$\mathrm{iO}_{3}$, , Philos. Mag. A., 60 [5] 601-12 (1989).

${ }^{9}$ A. Reènik and D. Kolar, "Exaggerated Growth of Hexagonal Barium Titanate under Reducing Sintering Conditions," J. Am. Ceram. Soc., 79 [4] 1015-8 (1996).

${ }^{10}$ D. B. Williams and C. B. Carter, Transmission Electron Microscopy-A Textbook for Materials Science, p. 381. Plenum, New York, 1996.

${ }^{11} \mathrm{~S}$. Amelinckx and J. van Landuyt, "The Study of Planar Interfaces by Means of Electron Microscopy"; pp. 107-51 in Diffraction and Imaging Techniques in Materials Science, Edited by S. Amelinckx, R. Gevers, and J. van Landuyt. NorthHolland, Amsterdam, 1978.

${ }^{12}$ P. A. Carvalho, M. Sijbolts, B. J. Kooi, and J. Th M. De Hosson, "High Resolution Transmission Electron Microscopy Study of Discontinuously Precipitated $\mathrm{Ni}_{3} \mathrm{Sn}, "$ Acta Mater., 48 [12] 4203-15 (2000).

${ }^{13}$ Y. C. Wu and H. Y. Lu, " $\pi$-Rotation Faults in Metastably Retained Hexagonal $\mathrm{BaTiO}_{3}$," J. Am. Ceram. Soc., 87 [9] 1753-63 (2004).

${ }^{14}$ A. H. Heuer, "Dislocations in $\alpha-\mathrm{Al}_{2} \mathrm{O}_{3}$ "; pp. 238-57 in Sintering of $\mathrm{Al}_{2} \mathrm{O}_{3}$ and $\mathrm{MgO}$, Advanced Ceramics, Vol. 10, Edited by W. D. Kingery. American Ceramic Society, Columbus, OH, 1984.

${ }^{15}$ M. H. Lin and H. Y. Lu, "Collinear Partial Dislocations in Barium Titanate Perovskite,” Mater. Sci. Eng., A333 [1-2] 41-4 (2002).

${ }^{16}$ Y. C. Wu, S. F. Wang, and H. Y. Lu. "Glide and Climb of Half Partial Dislocations in the Planar Fault Plane of Hexagonal Barium Titanate," unpublished results. 
Copyright of Journal of the American Ceramic Society is the property of Blackwell Publishing Limited and its content may not be copied or emailed to multiple sites or posted to a listserv without the copyright holder's express written permission. However, users may print, download, or email articles for individual use. 